\title{
Different Clinical Characteristics and Impacts of Carbapenem-Resistance on Outcomes Between Acinetobacter Baumannii and Pseudomonas Aeruginosa Bacteraemia: A Multicentre Prospective Observational Study
}

\section{Chan Mi Lee}

Seoul National University Bundang Hospital

\section{Young-Jun Kim}

Wonkwang University Hospital: Wonkwang University School of Medicine Hospital

\section{Sook-In Jung}

Chonnam National University Medical School

\section{Seong Eun Kim}

Chonnam National University Medical School

\section{Wan Beom Park}

Seoul National University Hospital

\section{Pyoeng Gyun Choe}

Seoul National University Hospital

\section{Eu Suk Kim}

Seoul National University Bundang Hospital

\section{Chung-Jong Kim}

Ewha Womans University Dongdaemun Hospital: Ewha Women's University Mokdong Hospital Hee Jung Choi

Ewha Womans University Dongdaemun Hospital: Ewha Women's University Mokdong Hospital

\section{Shinwon Lee}

Pusan National University Hospital

\section{Sun Hee Lee}

Pusan National University Hospital

\section{Younghee Jung}

Hallym University Sacred Heart Hospital

\section{Ji Hwan Bang}

Seoul Metropolitan Boramae Hospital: Seoul National University Seoul Metropolitan Government Boramae Medical Center

\section{Shinhye Cheon}


Chungnam National University Hospital

\section{Yee Gyung Kwak}

Inje University Ilsan Paik Hospital

\section{Yu Min Kang}

Kangwon National University Hospital

\section{Kyung-Hwa Park}

Chonnam National University Medical School

\section{Kyoung-Ho Song ( $\nabla$ khsongmd@gmail.com )}

Seoul National University Bundang Hospital https://orcid.org/0000-0002-4517-3840

\section{Hong Bin Kim}

Seoul National University Bundang Hospital

\section{Research}

Keywords: Carbapenem resistance, bacteraemia, risk factors, Acinetobacter baumannii, Pseudomonas aeruginosa

Posted Date: August 18th, 2021

DOI: https://doi.org/10.21203/rs.3.rs-812345/v1

License: (c) (i) This work is licensed under a Creative Commons Attribution 4.0 International License. Read Full License 


\section{Abstract}

Background: Carbapenem-resistance (CR) causes poor clinical outcomes and has limited treatment options. We aim to evaluate the differences in clinical characteristics and impact of carbapenemresistance on outcomes between Acinetobacter baumannii (ABA) and Pseudomonas aeruginosa (PAE) bacteraemia.

Methods: We prospectively identified all patients with ABA and PAE bacteraemia in 10 hospitals over a 1year period and collected their detailed clinical information. Treatment failure was defined as all-cause 30-day mortality, persistent bacteraemia, or recurrence within 30 days.

Results: We included 304 ABA and 241 PAE bacteraemia cases. CR was detected in 216 ABA (71\%) and 55 PAE (23\%). Treatment failure was significantly higher in CR-ABA than in CR-PAE $(60.6 \%$ vs. 34.5\%, $P=$ 0.001 ). Multivariate analyses were stratified by patient data according to $C R$ and the appropriateness of empirical therapy. For ABA patients, severe sepsis or septic shock and high Pitt bacteraemia score were independent risk factors for treatment failure in the inappropriate empirical antibiotics group. Pneumonia was a significant risk factor in the appropriate group. For PAE patients, hospital-acquired infection and high Pitt bacteraemia score were independent risk factors for treatment failure in both groups. CR was an independent risk factor in ABA for treatment failure in both the inappropriate (adjusted odds ratio [aOR]: $6.17,95 \%$ confidence interval [Cl]: 1.13-33.75, $P=0.036$ ) and appropriate empirical treatment groups (aOR: $4.15,95 \% \mathrm{Cl}: 1.16-14.84, P=0.029$ ), but not for patients with PAE bacteraemia.

Conclusions: We demonstrated significant differences in the clinical characteristics and impact of CR on the clinical outcomes between ABA and PAE bacteraemia. Our findings suggest that different approaches may be needed to treat ABA and PAE bacteraemia.

\section{Background}

Antimicrobial resistance is a major threat to public health. Recent reports from the Centers for Disease Control and Prevention concluded that the threat of antibiotic-resistance was greater than previously understood, and listed carbapenem resistance in non-fermenting gram negative bacteria, such as Acinetobacter baumannii (ABA) and Pseudomonas aeruginosa (PAE), as urgent threats (1). There are limited treatment options for carbapenem resistance; due to its severity and poor clinical outcomes, it is an emerging public health concern (2-4). Therefore, to further improve treatment for infectious diseases that show resistance to antibiotics, identifying their clinical features is critical.

Both carbapenem-resistant ABA (CR-ABA) and carbapenem-resistant PAE (CR-PAE) are important causes of healthcare-associated infections (5). Further, it is known that antibiotic susceptibility and clinical features vary between them. However, there are no integrated observational clinical studies assessing the clinical impacts of carbapenem resistance in ABA and PAE. Due to the limited treatment options for CR$A B A$ and $C R-P A E$, it is important to elucidate the impact of carbapenem resistance on the clinical outcomes and risk factors for treatment failure. Therefore, we aimed to evaluate the differences in the 
clinical characteristics and impacts of carbapenem resistance on clinical outcomes between ABA and PAE bacteraemia.

\section{Methods}

\section{Patient population and clinical data}

All patients with ABA or PAE bacteraemia were identified prospectively from clinical microbiology laboratories in 10 hospitals over a 1-year period from 1 September 2017 to 31 August 2018. We prospectively collected patients' demographic and microbiological data, underlying medical conditions, source of bacteraemia, clinical severity, antibiotic therapy, and clinical outcomes. This study was approved by the Institutional Review Board (IRB) of Seoul National University Bundang Hospital (IRB no. B-1804-463-105) and the IRBs of each participating hospital. The study was conducted in accordance with the principles outlined in the 1964 Declaration of Helsinki and its later amendments. The need for informed consent was waived by the IRBs.

\section{Definitions}

Mixed bacteraemia was defined as the isolation of other organisms except common skin flora, such as coagulase-negative staphylococci (6), from the first blood culture that yielded PAE or ABA. Bacteraemia detected in patients who were hospitalised for over 48 hours was classified as a hospital-acquired infection (HAl). Healthcare-associated infection was defined as fulfilling at least one of the following criteria: (1) hospitalisation for 2 or more days in the previous 12 months; (2) residence in a nursing home in the previous 12 months; (3) treatment with parenteral antibiotics within 30 days; and (4) renal replacement therapy within 30 days. Use of immunosuppressant was defined as the use of any anticancer chemotherapy, corticosteroids more than $20 \mathrm{mg}$ of prednisolone equivalent over 1 week, or any other immunosuppressive agent within the past 30 days.

The severity of illness was assessed using the Pitt bacteraemia score (7), and the severity of underlying disease was assessed using Charlson's weighted index of comorbidity (WIC) (8). Empirical antimicrobial therapy was defined as initial treatment used more than 24 hours after the sampling of the first positive blood culture. Antibiotic therapy was considered inappropriate if the isolate was not susceptible to the treatment regimen in vitro.

Treatment failure was defined as fulfilling any of the following events: (1) all-cause 30-day mortality; (2) persistent bacteraemia defined as positive blood cultures after $\geq 7$ days of appropriate antimicrobial therapy; (3) recurrent bacteraemia within 30 days after discontinuation of appropriate antimicrobial therapy (9).

\section{Data management and statistical analysis}


The chi-squared test or Fisher's exact test were used to compare categorical variables, and the Student ttest or Mann Whitney $\mathrm{U}$ test were used to compare continuous variables. A stepwise multiple logistic regression was used to identify independent risk factors for treatment failure. Risk factors with a $P$ value of $<0.10$ in univariate analysis and other variables of clinical significance were included in multivariate analysis. To avoid multicollinearity, severe sepsis or septic shock and Pitt bacteraemia score were analysed in a separate multivariate model. All underlying diseases and Charlson's WIC were analysed in a separate multivariate model for the same reason. $P$ values $<0.05$ were considered statistically significant. The Hosmer-Lemeshow test was used to assess the goodness of fit for logistic regression models. All statistical analyses were performed using IBM SPSS Statistics, version 26.0 (IBM Corp., Armonk, NY, USA).

\section{Results}

\section{Clinical characteristics of ABA and PAE bacteraemia}

Of the 545 cases identified during the 1-year study period, 304 were ABA bacteraemia and 241 were PAE bacteraemia. Of the 304 ABA bacteraemia cases, 216 (71\%) were CR-ABA and 88 (29\%) were carbapenem-susceptible ABA (CS-ABA). Of the 241 PAE bacteraemia cases, $55(23 \%)$ were CR-PAE and 186 (77\%) were carbapenem-susceptible PAE (CS-PAE).

The clinical characteristics of ABA bacteraemia according to carbapenem susceptibility are described in Table S1. Catheter related bloodstream infection and pneumonia were more frequent in the CR-ABA group than in the CS-ABA group $(42.1 \%$ vs. $28.4 \%, P=0.026 ; 36.1 \%$ vs. $9.1 \%, P<0.001)$. The CR-ABA group showed significantly higher percentages of severe sepsis or septic shock ( $53.2 \%$ vs. $21.6 \%, P<0.001)$ and inappropriate empirical therapy $(75.0 \%$ vs. $19.3 \%, P<0.001)$ than the CS-ABA group. All-cause 30 -day mortality and treatment failure rates were significantly higher in the CR-ABA group than the CS-ABA group (57.5\% vs. $15.5 \%, P<0.001 ; 60.6 \%$ vs. $15.9 \%, P<0.001)$.

The clinical characteristics of PAE bacteraemia according to carbapenem susceptibility are described in Table S2. The CR-PAE group showed significantly higher percentages of HAI $(61.8 \%$ vs. $45.2 \%, P=0.030)$ and underlying liver disease $(25.5 \%$ vs. $14.0 \%, P=0.044)$ than the CS-PAE group. The CR-PAE group showed significantly higher percentages of inappropriate empirical therapy than the CS-PAE group (52.7\% vs. $26.3 \%, P<0.001)$. There was no significant difference in treatment failure between the CR-PAE and CS-PAE groups ( $34.5 \%$ vs. $24.7 \%, P=0.150)$.

Comparisons of clinical characteristics of the CR-ABA and CR-PAE groups are shown in Table 1. The CRABA group showed significantly higher percentages of severe sepsis or septic shock than the CR-PAE group. Inappropriate empirical therapy was more frequent in the CR-ABA group than the CR-PAE group. All-cause 30-day mortality and treatment failure were significantly higher in the CR-ABA group than the CR-PAE group. 
Clinical characteristics of patients with carbapenem-resistant Acinetobacter baumannii (CR-ABA) and carbapenem-resistant Pseudomonas aeruginosa (CR-PAE) bacteraemia 


\begin{tabular}{|c|c|c|c|c|}
\hline Variables & $\begin{array}{l}\text { Total } \\
(n=271)\end{array}$ & $\begin{array}{l}\text { CR-ABA } \\
(n=216)\end{array}$ & $\begin{array}{l}\text { CR-PAE } \\
(n=55)\end{array}$ & $P$ \\
\hline Age, mean ( $\pm S D)$ & $65.6( \pm 16.9)$ & $66.0( \pm 17.2)$ & $64.1( \pm 15.4)$ & 0.469 \\
\hline Male & $177(65.3)$ & $143(66.2)$ & $34(61.8)$ & 0.542 \\
\hline \multicolumn{5}{|l|}{ at bacteraemia onset } \\
\hline Hospital-acquired infection & $218(80.4)$ & $184(85.2)$ & $34(61.8)$ & $<0.001$ \\
\hline Healthcare-associated infection & $260(95.9)$ & $211(97.7)$ & $49(89.1)$ & 0.004 \\
\hline Mixed bacteraemia & $29(10.7)$ & $18(8.3)$ & $11(20.0)$ & 0.012 \\
\hline \multicolumn{5}{|l|}{ Site of infection } \\
\hline Primary bacteraemia & $76(28.0)$ & $58(26.9)$ & $18(32.7)$ & 0.387 \\
\hline CRBSI & $103(38.0)$ & $91(42.1)$ & $12(21.8)$ & 0.006 \\
\hline Pneumonia & $82(30.3)$ & $78(36.1)$ & $4(7.3)$ & $<0.001$ \\
\hline Urinary tract infection & $55(20.3)$ & $45(20.8)$ & $10(18.2)$ & 0.662 \\
\hline Intra-abdominal infection & $26(9.6)$ & $16(7.4)$ & $10(18.2)$ & 0.015 \\
\hline \multicolumn{5}{|l|}{ Underlying medical condition } \\
\hline Charlson's WIC $\geq 3$ & $216(79.7)$ & $172(79.6)$ & $44(80.0)$ & 0.951 \\
\hline Heart disease & $28(10.3)$ & $24(11.1)$ & $4(7.3)$ & 0.404 \\
\hline Lung disease & $27(10.0)$ & $26(12.0)$ & $1(1.8)$ & 0.024 \\
\hline Chronic kidney disease & $51(18.8)$ & $43(19.9)$ & $8(14.5)$ & 0.364 \\
\hline Liver disease & $56(20.7)$ & $42(19.4)$ & $14(25.5)$ & 0.326 \\
\hline Diabetes mellitus & $79(29.2)$ & $69(31.9)$ & $10(18.2)$ & 0.045 \\
\hline Malignancy & $78(28.8)$ & $55(25.5)$ & $23(41.8)$ & 0.017 \\
\hline Cerebrovascular disease & $59(21.8)$ & $48(22.2)$ & $11(20.0)$ & 0.721 \\
\hline Transplantation & $16(5.9)$ & $11(5.1)$ & $5(9.1)$ & 0.332 \\
\hline Immunosuppressant use & $37(13.7)$ & $28(13.0)$ & $9(16.4)$ & 0.512 \\
\hline \multicolumn{5}{|l|}{ Clinical severity } \\
\hline Severe sepsis or septic shock & $136(50.2)$ & $115(53.2)$ & $21(38.2)$ & 0.046 \\
\hline Pitt score, median (IQR) & $\begin{array}{l}4.00(2.00- \\
6.00)\end{array}$ & $\begin{array}{l}5.00(2.00- \\
7.00)\end{array}$ & $\begin{array}{l}2.00(1.00- \\
4.00)\end{array}$ & $<0.001$ \\
\hline
\end{tabular}




\begin{tabular}{|lcccc|}
\hline $\begin{array}{l}\text { Inappropriate empirical } \\
\text { antibiotics }\end{array}$ & $191(70.5)$ & $162(75.0)$ & $29(52.7)$ & $\mathbf{0 . 0 0 1}$ \\
\hline All-cause 30-day mortality & $135(51.1)$ & $122(57.5)$ & $13(25.0)$ & $<0.001$ \\
\hline Treatment failure & $150(55.4)$ & $131(60.6)$ & $19(34.5)$ & $\mathbf{0 . 0 0 1}$ \\
\hline $\begin{array}{l}\text { SD, standard deviation; ICU, intensive care unit; CRBSI, catheter-related bloodstream infection; WIC, } \\
\text { weighted index of comorbidity; IQR, interquartile range }\end{array}$ & \\
\hline
\end{tabular}

\section{Antibiotic susceptibility of carbapenem-resistant isolates}

Antibiotic susceptibility of carbapenem-resistant isolates is described in Table 2. Not all results of susceptibility tests were available for carbapenem-resistant isolates. For all antibiotics except colistin and trimethoprim/sulfamethoxazole, the percentages of non-susceptible isolates were significantly different between the CR-ABA and CR-PAE groups. The percentages of non-susceptible isolates, except for tigecycline and minocycline, were significantly higher in the CR-ABA group than the CR-PAE group.

\section{Table 2}

Antibiotic susceptibility of carbapenem-resistant Acinetobacter baumannii (CR-ABA) and carbapenemresistant Pseudomonas aeruginosa (CR-PAE) isolates 


\begin{tabular}{|lllll|}
\hline Non-susceptible (I+R) isolate No. $(\%)$ & $\begin{array}{l}\text { Total* } \\
(\mathrm{n}=271)\end{array}$ & $\begin{array}{l}\text { CR-ABA } \\
(\mathrm{n}=216)\end{array}$ & $\begin{array}{l}\text { CR-PAE } \\
(\mathrm{n}=55)\end{array}$ & $P$ \\
\hline Ceftazidime & $240 / 268(89.6)$ & $209(97.2)$ & $31(58.5)$ & $<0.001$ \\
\hline Cefepime & $242 / 265(91.3)$ & $211(98.6)$ & $31(60.8)$ & $<0.001$ \\
\hline Ciprofloxacin & $239 / 269(88.8)$ & $210(97.2)$ & $29(54.7)$ & $<0.001$ \\
\hline Amikacin & $66 / 130(50.8)$ & $58(70.7)$ & $8(16.7)$ & $<0.001$ \\
\hline Gentamicin & $175 / 245(71.4)$ & $161(77.8)$ & $14(36.8)$ & $<0.001$ \\
\hline Tobramycin & $45 / 88(51.1)$ & $40(74.1)$ & $5(14.7)$ & $<0.001$ \\
\hline Piperacillin/tazobactam & $225 / 243(92.6)$ & $191(99.0)$ & $34(68.0)$ & $<0.001$ \\
\hline Trimethoprim/sulfamethoxazole & $145 / 174(83.3)$ & $134(82.2)$ & $11(100.0)$ & 0.215 \\
\hline Imipenem & $252 / 260(96.9)$ & $211(99.1)$ & $41(87.2)$ & 0.001 \\
\hline Meropenem & $254 / 261(97.3)$ & $213(99.5)$ & $41(87.2)$ & $<0.001$ \\
\hline Tigecycline & $24 / 118(20.3)$ & $16(14.5)$ & $8(100.0)$ & $<0.001$ \\
\hline Colistin & $4 / 217(1.8)$ & $3(1.7)$ & $1(2.7)$ & 0.529 \\
\hline Minocycline & $47 / 174(27.0)$ & $38(23.2)$ & $9(90.0)$ & $<0.001$ \\
\hline $\begin{array}{l}\text { I, intermediate; } \text { R, resistant; } * \text { Not all isolate susceptibility test results were available, and results were } \\
\text { presented as non-susceptible isolate number/available total (\%). }\end{array}$ & & \\
\hline
\end{tabular}

\section{Risk factors for treatment failure}

The results of univariate analysis between risk factors and treatment failure in ABA are listed in Table S3. We stratified the patients' data according to carbapenem resistance and the appropriateness of empirical therapy in the multivariate analyses of risk factors for treatment failure, because of the significant interaction between those factors.

Table 3 shows the risk factors for treatment failure in ABA identified using multivariate analysis according to the appropriateness of empirical therapy. While severe sepsis or septic shock and high Pitt bacteraemia score were independent risk factors for treatment failure in the inappropriate empirical antibiotics group, pneumonia, and Charlson's WIC $\geq 3$ were independent risk factors for treatment failure in the appropriate empirical antibiotics group.

Table 3 
Risk factors for treatment failure in patients with Acinetobacter baumannii bacteraemia, according to the appropriateness of empirical therapy

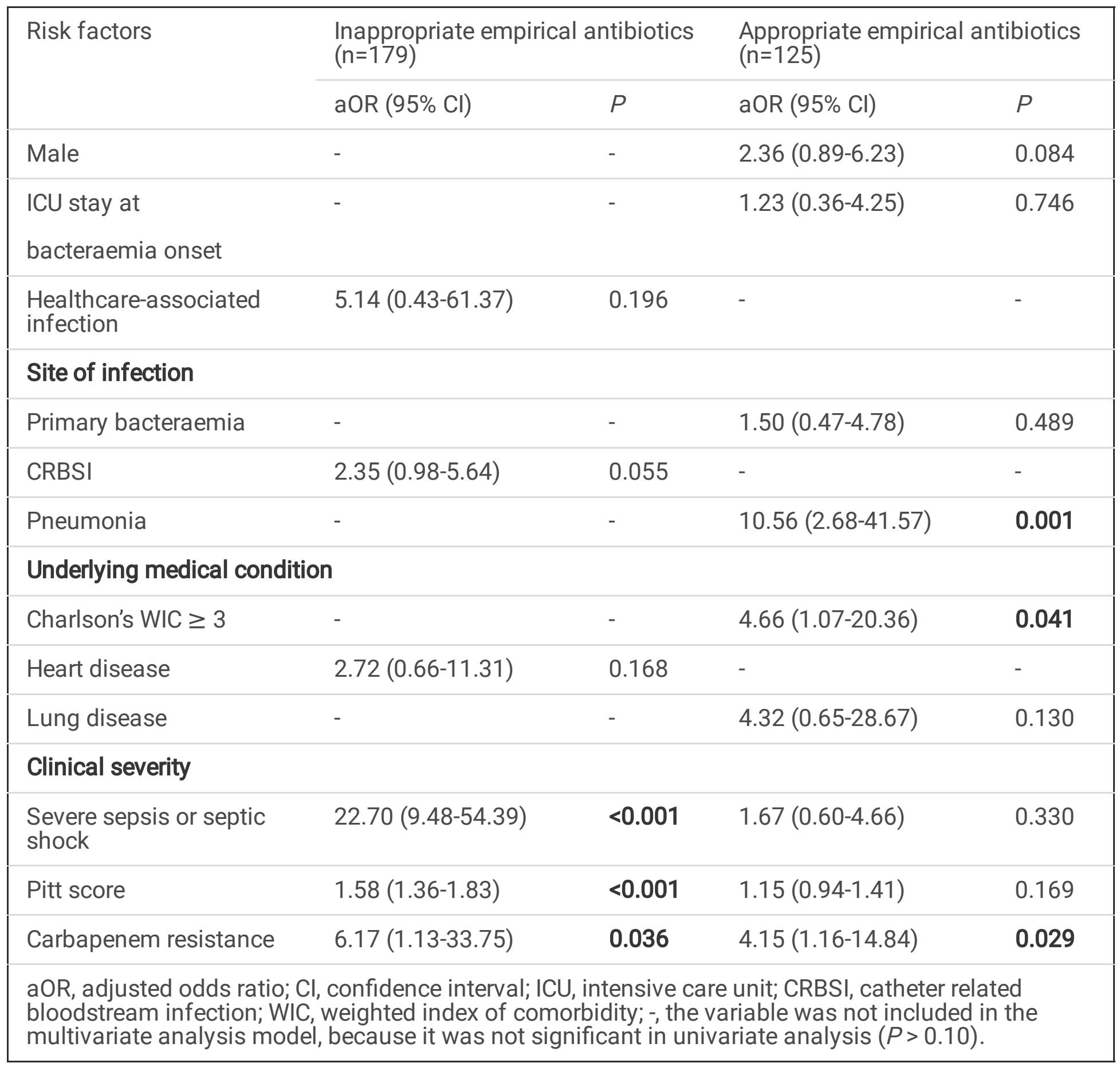

The results of multivariate analysis between risk factors and treatment failure in $A B A$ according to carbapenem susceptibility are listed in Table S4. In the CR-ABA group, severe sepsis or septic shock (adjusted odds ratio [aOR]: 9.70, 95\% confidence interval [CI]: 5.02-18.76, $P<0.001$ ) and high Pitt bacteraemia score (aOR: $1.44,95 \% \mathrm{Cl}: 1.27-1.63, P<0.001)$ were significantly associated with treatment failure.

The results of univariate analysis between risk factors and treatment failure in PAE bacteraemia are listed in Table S5. Significant risk factors for treatment failure in PAE identified by multivariate analysis are 
listed by the appropriateness of empirical therapy in Table 4. HAl, severe sepsis or septic shock, and high Pitt score were independent risk factors for treatment failure in both the inappropriate and appropriate empirical antibiotics groups. Urinary tract infection was associated with decreased treatment failure in the appropriate antibiotics group. Table $\mathrm{S} 6$ shows the significant risk factors for treatment failure in PAE by multivariate analysis according to carbapenem susceptibility. In the CR-PAE group, HAI (aOR: 25.03, 95\% Cl: 2.15-291.99, $P=0.010$ ), use of immunosuppressant (aOR: 32.08, 95\% Cl: 1.58-652.62, $P=0.024$ ), and high Pitt bacteraemia score (aOR: $1.47,95 \% \mathrm{Cl}: 1.05-2.07, P=0.025)$ were significantly associated with treatment failure.

\section{Carbapenem resistance and treatment failure}

In $A B A$, carbapenem resistance was independently associated with treatment failure in both the inappropriate (aOR: $6.17 ; 95 \% \mathrm{Cl}: 1.13-33.75 ; P=0.036$ ) and appropriate empirical treatment groups (aOR: $4.15 ; 95 \% \mathrm{Cl}: 1.16-14.84 ; P=0.029$; Table 3 ). In PAE, carbapenem resistance was not significantly associated with treatment failure in both the inappropriate (aOR: $0.91,95 \% \mathrm{Cl}: 0.27-3.03, P=0.879$ ) and appropriate empirical antibiotics groups (aOR: 1.76, 95\% Cl: 0.45-6.87, $P=0.418$; Table 4).

\section{Table 4}

Risk factors for treatment failure in patients with Pseudomonas aeruginosa bacteraemia, according to the appropriateness of empirical therapy 


\begin{tabular}{|c|c|c|c|c|}
\hline \multirow[t]{2}{*}{ Risk factors } & \multicolumn{2}{|c|}{$\begin{array}{l}\text { Inappropriate empirical antibiotics } \\
(\mathrm{n}=78)\end{array}$} & \multicolumn{2}{|c|}{$\begin{array}{l}\text { Appropriate empirical antibiotics } \\
(\mathrm{n}=163)\end{array}$} \\
\hline & aOR $(95 \% \mathrm{Cl})$ & $P$ & aOR $(95 \% \mathrm{Cl})$ & $P$ \\
\hline Male & - & - & $3.61(1.23-10.65)$ & 0.020 \\
\hline \multicolumn{4}{|l|}{ at bacteraemia onset } & 0.645 \\
\hline $\begin{array}{l}\text { Hospital-acquired } \\
\text { infection }\end{array}$ & $4.57(1.34-15.54)$ & 0.015 & $2.88(1.09-7.65)$ & 0.033 \\
\hline \multicolumn{5}{|l|}{ Site of infection } \\
\hline Primary bacteraemia & - & - & $2.79(0.94-8.25)$ & 0.064 \\
\hline Pneumonia & $4.61(0.68-31.20)$ & 0.117 & $3.82(1.09-13.32)$ & 0.036 \\
\hline Urinary tract infection & - & - & $0.13(0.02-0.79)$ & 0.027 \\
\hline \multicolumn{5}{|c|}{ Underlying medical condition } \\
\hline Malignancy & $2.09(0.55-7.91)$ & 0.276 & $1.81(0.66-4.96)$ & 0.251 \\
\hline $\begin{array}{l}\text { Immunosuppressant } \\
\text { use }\end{array}$ & $3.31(0.78-14.11)$ & 0.105 & $1.58(0.54-4.63)$ & 0.406 \\
\hline \multicolumn{5}{|l|}{ Clinical severity } \\
\hline $\begin{array}{l}\text { Severe sepsis or septic } \\
\text { shock }\end{array}$ & $5.43(1.42-20.76)$ & 0.013 & $10.33(3.56-29.94)$ & $<0.001$ \\
\hline Pitt score & $1.59(1.21-2.10)$ & 0.001 & $1.59(1.27-1.99)$ & $<0.001$ \\
\hline Carbapenem resistance & $0.91(0.27-3.03)$ & 0.879 & $1.76(0.45-6.87)$ & 0.418 \\
\hline
\end{tabular}

aOR, adjusted odds ratio; $\mathrm{Cl}$, confidence interval; ICU, intensive care unit

\section{Discussion}

Resistance to carbapenem is an important concern in both ABA and PAE bacteraemia. Here, we found different clinical characteristics and impacts of carbapenem-resistance on outcomes in ABA and PAE bacteraemia.

Carbapenem resistance was an independent risk factor for treatment failure in ABA bacteraemia regardless of the appropriateness of empirical antibiotics. This finding agrees with previous studies, which report that carbapenem resistance is significantly associated with mortality in ABA bacteraemia $(10,11)$. A systematic review showed that carbapenem resistance may increase the risk of mortality of ABA infection (12). In contrast, we found that carbapenem resistance was not an independent risk factor for treatment failure in PAE bacteraemia regardless of the appropriateness of empirical antibiotics. This 
is in agreement with a previous study showing that carbapenem resistance was not an independent risk factor for mortality in PAE bacteraemia (13). This difference in the impact of carbapenem-resistance on ABA and PAE may be due to available treatment options, different sites of infection, and differences of clinical severity.

We found different susceptibilities and clinical characteristics in ABA and PAE bacteraemia. In ABA bacteraemia, $71.1 \%(216 / 304)$ of the cases were carbapenem resistant, while only $22.8 \%(55 / 241)$ of the cases in PAE bacteraemia were carbapenem resistant. The higher percentage of carbapenem resistance in ABA compared with PAE bacteraemia is consistent with previous results, which showed that the proportion of carbapenem resistance was $92.1 \%$ in ABA strains and the proportions of resistance were $19.5 \%$ to imipenem and $18.1 \%$ to meropenem in PAE strains (14). We found that CR-ABA have resistance to almost all antibiotics; however, CR-PAE is susceptible to some antibiotics such as ciprofloxacin and amikacin. The difference in antibiotic susceptibility might explain the difference in the proportion of inappropriate empirical therapies between the groups. However, the results of multivariate analysis using stratification to the appropriateness of empirical therapy showed that carbapenem resistance is an independent risk factor for treatment failure in $A B A$, even if the empirical therapy was appropriate. This might be related to the virulence of $A B A$. A previous study suggested that high bacterial cytotoxicity significantly affected the mortality in ABA ventilator-associated pneumonia (15).

Treatment failure was not significantly different between CR-PAE and CS-PAE, whereas it was significantly different between CR-ABA and CS-ABA. Moreover, a previous study found a significant difference in hospital mortality between CR-ABA and CS-ABA, but not between CR-PAE and CS-PAE (16). Compared to the CR-PAE group, the CR-ABA group showed a significantly higher percentage of treatment failure $(60.6 \%$ vs. $34.5 \%)$. The different clinical severity and antibiotics susceptibility between CR-ABA and CR-PAE might contribute to the differences in treatment failure. Clinicians should consider the higher rates of treatment failure when carbapenem resistance is detected in ABA.

In ABA bacteraemia, we found independent risk factors, including pneumonia, Charlson's WIC $\geq 3$, and carbapenem resistance in the appropriate empirical antibiotics group. We speculate that if pneumonia was the source of bacteraemia, or if carbapenem resistance was detected in ABA bacteraemia, the risk of treatment failure would be high, even if appropriate empirical antibiotics were administered. One study identified independent risk factors for mortality, including carbapenem resistance, neutropenia, and prolonged intensive care unit stay in ABA bacteraemia (10). Another study showed that septic shock, carbapenem resistance, pneumonia, and inappropriate definite antimicrobial therapy are independent risk factors for 14-day mortality (17).

Independent risk factors for treatment failure in PAE bacteraemia included HAl, severe sepsis or septic shock, and high Pitt bacteraemia score, regardless of the appropriateness of empirical antibiotics. One retrospective study reported the presence of septic shock and high Acute Physiologic Assessment and Chronic Health Evaluation II score as independent risk factors for 14-day mortality (13). Another study showed that recent hospitalisation, corticosteroid treatment, Charlson WIC, non-urinary source, and high 
Sequential Organ Failure Assessment score are significant risk factors for 30-day mortality in PAE bacteraemia (18).

By additional analysis according to carbapenem resistance, we found that inappropriate empirical antibiotics were independently associated with treatment failure in the CS-PAE group. Appropriate empirical antibiotics, including antipseudomonal coverage, may be important in the management of patients with risk of Pseudomonas infection. The independent risk factors for treatment failure in the CRPAE group were HAl, use of immunosuppressants, and high Pitt bacteraemia score. This is consistent with a previous study reporting that high Pitt bacteraemia score is a predictor of death in CR-PAE bacteraemia (19).

In both the CR-ABA and CS-ABA groups, inappropriate empirical antibiotics was not an independent factor for treatment failure. Furthermore, a previous study found that 28-day mortality was not independently associated with appropriate empirical antimicrobial therapy in invasive Acinetobacter infection (20). Although studies found that appropriate antimicrobial therapy decreased 14-day mortality $(21,22)$, these defined appropriate antimicrobial therapy as antibiotics administration within 48 hours after the onset of bacteraemia and did not address the appropriateness of empirical antibiotics.

This study has several limitations. First, being an observational study, it was prone to bias and unmeasured confounding factors. Second, no microbiologic investigation other than antibiotic susceptibility tests was conducted on the strains. As the effect of carbapenemase on clinical outcomes is not clear, further studies on the effect of the microbiological characteristics on the outcome in ABA and PAE are needed. Lastly, while colistin was the only drug available in Korea for pandrug-resistant $A B A$ and difficult-to-treat PAE at the time of the study, several new drugs, such as ceftolozane-tazobactam, ceftazidime-avibactam, imipenem-relebactam, and cefiderocol were available in other countries $(23,24)$. This difference in availability of drugs may have contributed to the differences in prognosis.

\section{Conclusions}

In conclusion, we identified clinical characteristics and risk factors associated with treatment failure in $A B A$ and PAE bacteraemia. Carbapenem resistance was associated with increased risk of treatment failure in ABA, but not in PAE bacteraemia. Because carbapenem resistance is significantly associated with treatment failure in the appropriate empirical antibiotics group with ABA, this study suggests that appropriate empirical antibiotics cannot improve the clinical outcomes in CR-ABA. Hence, we suggest that it would be important to reduce the occurrence of CR-ABA through infection control in high-risk groups such as patients in intensive care units.

\section{Declarations}

\section{Ethics approval and consent to participate}


This study was approved by the Institutional Review Board (IRB) of Seoul National University Bundang Hospital (IRB no. B-1804-463-105) and the IRBs of each participating hospital. The study was conducted in accordance with the principles outlined in the 1964 Declaration of Helsinki and its later amendments. The need for informed consent was waived by the IRBs.

\section{Consent for publication}

Not applicable.

\section{Availability of data and materials}

The data that support the findings of this study are available from the corresponding author, KHS, upon reasonable request.

\section{Competing interests}

The authors declare that they have no competing interests.

\section{Funding}

This work was supported by the Research Program funded by the Korea Disease Control and Prevention Agency (2017E280301). This research was also supported by the National Research Foundation of Korea (NRF) grant funded by the Korea government (MSIT) (No. 2021R1F1A1063089).

\section{Author contributions}

KHS and KHP conceptualized the study. CML and YJK analyzed the data and drafted the initial manuscript. SIJ, SEK, WBP, PGC, ESK, CJK, HJC, SL, SHL, YJ, JHB, SC, YGK, YMK and HBK collected patient data and made contributions to the manuscript. KHS and HBK administrated the project. KHS and KHP revised and edited the final manuscript. All authors approved the final manuscript for publication.

\section{References}

1. CDC. Antibiotic Resistance Threats in the United States. 2019. Atlanta, GA: US Department of Health and Human Services, CDC. 2019.

2. Logan LK, Weinstein RA. The Epidemiology of Carbapenem-Resistant Enterobacteriaceae: The Impact and Evolution of a Global Menace. J Infect Dis. 2017 Feb 15;215(suppl_1):S28-S36. PubMed PMID: 28375512. Pubmed Central PMCID: PMC5853342. Epub 2017/04/05. 
3. Zilberberg MD, Nathanson BH, Sulham K, Fan W, Shorr AF. Carbapenem resistance, inappropriate empiric treatment and outcomes among patients hospitalized with Enterobacteriaceae urinary tract infection, pneumonia and sepsis. BMC Infect Dis. 2017 Apr 17;17(1):279. PubMed PMID: 28415969. Pubmed Central PMCID: PMC5393012. Epub 2017/04/19.

4. Durante-Mangoni E, Andini R, Zampino R. Management of carbapenem-resistant Enterobacteriaceae infections. Clin Microbiol Infect. 2019 Aug;25(8):943-50. PubMed PMID: 31004767. Epub 2019/04/21.

5. Tomczyk S, Zanichelli V, Grayson ML, Twyman A, Abbas M, Pires D, et al. Control of Carbapenemresistant Enterobacteriaceae, Acinetobacter baumannii, and Pseudomonas aeruginosa in Healthcare Facilities: A Systematic Review and Reanalysis of Quasi-experimental Studies. Clin Infect Dis. 2019 Feb 15;68(5):873-84. PubMed PMID: 30475989. Pubmed Central PMCID: PMC6389314. Epub 2018/11/27.

6. Hall KK, Lyman JA. Updated review of blood culture contamination. Clin Microbiol Rev. 2006 Oct;19(4):788-802. PubMed PMID: 17041144. Pubmed Central PMCID: PMC1592696. Epub 2006/10/17.

7. Paterson DL, Ko WC, Von Gottberg A, Mohapatra S, Casellas JM, Goossens H, et al. International prospective study of Klebsiella pneumoniae bacteremia: implications of extended-spectrum betalactamase production in nosocomial Infections. Ann Intern Med. 2004 Jan;6(1):26-32. 140(. PubMed PMID: 14706969. Epub 2004/01/07.

8. Charlson ME, Pompei P, Ales KL, MacKenzie CR. A new method of classifying prognostic comorbidity in longitudinal studies: development and validation. J Chronic Dis. 1987;40(5):373-83. PubMed PMID: 3558716. Epub 1987/01/01.

9. Chen SY, Liao CH, Wang JL, Chiang WC, Lai MS, Chie WC, et al. Methicillin-resistant Staphylococcus aureus (MRSA) staphylococcal cassette chromosome mec genotype effects outcomes of patients with healthcare-associated MRSA bacteremia independently of vancomycin minimum inhibitory concentration. Clin Infect Dis. 2012 Nov 15;55(10):1329-37. PubMed PMID: 22911641. Epub 2012/08/23.

10. Choe YJ, Lee HJ, Choi EH. Risk Factors for Mortality in Children with Acinetobacter baumannii Bacteremia in South Korea: The Role of Carbapenem Resistance. Microb Drug Resist. 2019 Oct;25(8):1210-8. PubMed PMID: 31120349. Epub 2019/05/24.

11. Park SY, Choo JW, Kwon SH, Yu SN, Lee EJ, Kim TH, et al. Risk Factors for Mortality in Patients with Acinetobacter baumannii Bacteremia. Infect Chemother. 2013 Sep;45(3):325-30. PubMed PMID: 24396634. Pubmed Central PMCID: PMC3848511. Epub 2014/01/08.

12. Lemos EV, de la Hoz FP, Einarson TR, McGhan WF, Quevedo E, Castaneda C, et al. Carbapenem resistance and mortality in patients with Acinetobacter baumannii infection: systematic review and meta-analysis. Clin Microbiol Infect. 2014 May;20(5):416-23. PubMed PMID: 24131374. Epub 2013/10/18. 
13. Kim YJ, Jun YH, Kim YR, Park KG, Park YJ, Kang JY, et al. Risk factors for mortality in patients with Pseudomonas aeruginosa bacteremia; retrospective study of impact of combination antimicrobial therapy. BMC Infect Dis. 2014 Mar 24;14:161. PubMed PMID: 24655422. Pubmed Central PMCID: PMC3994322. Epub 2014/03/25.

14. Liu C, Yoon EJ, Kim D, Shin JH, Shin JH, Shin KS, et al. Antimicrobial resistance in South Korea: A report from the Korean global antimicrobial resistance surveillance system (Kor-GLASS) for 2017. J Infect Chemother. 2019 Nov;25(11):845-59. PubMed PMID: 31311694. Epub 2019/07/18.

15. Ju MH, Hou DN, Chen S, Wang Y, Tang XJ, Liu J, et al. Risk factors for mortality in ICU patients with Acinetobacter baumannii ventilator-associated pneumonia: impact of bacterial cytotoxicity. $J$ Thorac Dis. 2018 May;10(5):2608-17. PubMed PMID: WOS:000435834200031. English.

16. Zhen X, Stalsby Lundborg C, Sun X, Gu S, Dong H. Clinical and Economic Burden of CarbapenemResistant Infection or Colonization Caused by Klebsiella pneumoniae, Pseudomonas aeruginosa, Acinetobacter baumannii: A Multicenter Study in China. Antibiotics (Basel). 2020 Aug 13;9(8). PubMed PMID: 32823707. Pubmed Central PMCID: PMC7459498. Epub 2020/08/23.

17. Kim YJ, Kim SI, Hong KW, Kim YR, Park YJ, Kang MW. Risk factors for mortality in patients with carbapenem-resistant Acinetobacter baumannii bacteremia: impact of appropriate antimicrobial therapy. J Korean Med Sci. 2012 May;27(5):471-5. PubMed PMID: 22563209. Pubmed Central PMCID: PMC3342535. Epub 2012/05/09.

18. Babich T, Naucler P, Valik JK, Giske CG, Benito N, Cardona R, et al. Risk factors for mortality among patients with Pseudomonas aeruginosa bacteraemia: a retrospective multicentre study. Int $\mathrm{J}$ Antimicrob Agents. 2020 Feb;55(2):105847. PubMed PMID: 31770625. Epub 2019/11/27.

19. Buehrle DJ, Shields RK, Clarke LG, Potoski BA, Clancy CJ, Nguyen MH. Carbapenem-Resistant Pseudomonas aeruginosa Bacteremia: Risk Factors for Mortality and Microbiologic Treatment Failure. Antimicrob Agents Chemother. 2017 Jan;61(1). PubMed PMID: 27821456. Pubmed Central PMCID: PMC5192105. Epub 2016/11/09.

20. Kiyasu Y, Hitomi S, Funayama Y, Saito K, Ishikawa H. Characteristics of invasive Acinetobacter infection: A multicenter investigation with molecular identification of causative organisms. J Infect Chemother. 2020 May;26(5):475-82. PubMed PMID: 31924521. Epub 2020/01/12.

21. Lee YT, Kuo SC, Yang SP, Lin YT, Tseng FC, Chen TL, et al. Impact of appropriate antimicrobial therapy on mortality associated with Acinetobacter baumannii bacteremia: relation to severity of infection. Clin Infect Dis. 2012 Jul;55(2):209-15. PubMed PMID: 22495546. Epub 2012/04/13.

22. Kang FY, How CK, Wang YC, Cheng A, Yang YS, Kuo SC, et al. Influence of severity of infection on the effect of appropriate antimicrobial therapy for Acinetobacter baumannii bacteremic pneumonia. Antimicrob Resist Infect Control. 2020 Sep 29;9(1):160. PubMed PMID: 32993810. Pubmed Central PMCID: PMC7523485. Epub 2020/10/01.

23. Tamma PD, Aitken SL, Bonomo RA, Mathers AJ, van Duin D, Clancy CJ. Infectious Diseases Society of America Guidance on the Treatment of Extended-Spectrum beta-lactamase Producing Enterobacterales (ESBL-E), Carbapenem-Resistant Enterobacterales (CRE), and Pseudomonas 
aeruginosa with Difficult-to-Treat Resistance (DTR-P. aeruginosa). Clin Infect Dis. $2021 \mathrm{Apr}$ 8;72(7):1109-16. PubMed PMID: 33830222. Epub 2021/04/09.

24. Doi Y. Treatment Options for Carbapenem-resistant Gram-negative Bacterial Infections. Clin Infect Dis. 2019 Nov 13;69(Suppl 7):S565-S75. PubMed PMID: 31724043. Pubmed Central PMCID: PMC6853760. Epub 2019/11/15.

\section{Supplementary Files}

This is a list of supplementary files associated with this preprint. Click to download.

- Additionalfile1.docx 